\title{
The first bismuth(III)-catalyzed guanylation of thioureas
}

\author{
Silvio Cunha* and Manoel T. Rodrigues, Jr. \\ Instituto de Química, Universidade Federal da Bahia, Campus de Ondina, Salvador-BA 40170-290, Brazil
}

Received 10 February 2006; accepted 26 July 2006

\begin{abstract}
This work describes the first catalytic bismuth-promoted synthesis of polysubstituted guanidines in good yields through the guanylation reaction of $N$-benzoyl or $N$-phenylthioureas with primary and secondary amines, but now employing equimolar amounts of each organic reagent. Both bismuth iodine and bismuth nitrate were efficient as inorganic thiophiles at only 5 mol $\%$ in relation to substrates, being the first example of inorganic thiophiles acting in guanylation at catalytic levels.
\end{abstract}

(c) 2006 Elsevier Ltd. All rights reserved.

Despite of the variety of guanylating reagents to solution synthesis of guanidines, the most used guanylation protocols employ protected thiourea as guanylating reagents and stoichiometric amounts of heavy metal salts as guanylating agent, $\mathrm{HgCl}_{2}$ being the toxic and the most popular.

Bismuth is the least toxic of the heavy metals and their salts have been intensively used in organic transformations. ${ }^{2,3}$ We have reported guanylation of $N$-benzoylthioureas using stoichiometric amounts of $\mathrm{Bi}\left(\mathrm{NO}_{3}\right)_{3}$ as thiophile, but the use of 2 equiv of the amine was required to achieve an yield comparable to the $\mathrm{HgCl}_{2}$ method. ${ }^{4}$ This is a serious drawback when expensive or no commercial amines are necessary. Herein, we describe the first successful catalytic Bi(III)-mediated synthesis of functionalized guanidines. Besides, no excess of nucleophilic amine was needed.

In the previously stoichiometric bismuth-promoted guanylation, we identified $\mathrm{Bi}_{2} \mathrm{~S}_{3}$ as byproduct. Here we hypothesized that stoichiometric amount of $\mathrm{Bi}\left(\mathrm{NO}_{3}\right)_{3}$ was necessary because the low solubility of $\mathrm{Bi}_{2} \mathrm{~S}_{3}$ in the reaction medium precluded the continuous thiourea activation, that is, necessary to the guanylation. Thus, we reason that a catalytic cycle could operate if $\mathrm{Bi}_{2} \mathrm{~S}_{3}$ could be in situ transformed into a more soluble $\mathrm{Bi}(\mathrm{III})$ derivative, affording guanidines in good yields. To this end, an oxidant agent as co-reagent could be useful

\footnotetext{
Keywords: Thioureas; Guanidines; Bismuth.

* Corresponding author. Tel.: +55 71 32375784; fax: +55 71

32374117; e-mail: silviodc@ufba.br
}

because $\left(\mathrm{SO}_{x}\right)^{y-}$-derivatives of $\mathrm{Bi}(\mathrm{III})$ are more soluble than $\mathrm{Bi}_{2} \mathrm{~S}_{3} \cdot{ }^{1 a}$ To test this hypothesis we reacted thiourea 2a with amine 3a in the presence of diverse Bi(III) salts and oxidants. After experimentation, $\mathbf{4 a}$ was obtained in $15 \%$ yield after 5 days when $\mathrm{BiI}_{3} 5 \mathrm{~mol} \%$ was used in the presence of $\mathrm{K}_{2} \mathrm{~S}_{2} \mathrm{O}_{8}$. The oxidant was thus screened. Changing the oxidant to sodium bismuthate, $\mathrm{NaBiO}_{3}$, guanidine $4 \mathrm{a}$ was isolated in $92 \%$ yield and $10 \mathrm{~h}$, Table 1 . The use of cheap $\mathrm{NaBiO}_{3}$ is noteworthy because both thiophile and oxidant are now environmental friendly. ${ }^{2,5}$ This protocol could be extended to less activated $\mathrm{N}$ phenylthioureas, and primary and secondary amines were tolerated as the nucleophilic component using both $\mathrm{N}$-substituted thioureas, affording a representative spectrum of guanidines through this new catalytic guanylation (Table 1). $\mathrm{Bi}\left(\mathrm{NO}_{3}\right)_{3} \quad 5 \mathrm{~mol} \%$ was also effective but $\mathrm{BiI}_{3}$ afforded better yield and shorter reaction time. For instance, guanylation of $\mathbf{2 a}$ with cyclohexylamine and $\mathrm{Bi}\left(\mathrm{NO}_{3}\right)_{3} 5 \mathrm{~mol} \%$ afforded $4 \mathbf{c}$ in $69 \%$ yield $(21 \mathrm{~h})$.

The proposed catalytic cycle for this guanylation is indicated in Figure 1. In analogy to the $\mathrm{HgCl}_{2}$-promoted guanylation, ${ }^{1}$ we assumed that a carbodiimide is the key intermediate. In this way, Bi(III) should behave as a thiophilic soft Lewis acid that coordinated to the thiourea followed by reaction with $\mathrm{Et}_{3} \mathrm{~N}$ and form the carbodiimide by thiourea desulfurization, which is trapped by the amine affording the guanidine, and the insoluble $\mathrm{Bi}_{2} \mathrm{~S}_{3}$ is oxidized to a more soluble $\mathrm{Bi}(\mathrm{III})$ derivative.

In conclusion, we have developed the first catalytic $\mathrm{Bi}(\mathrm{III})$ guanylation of thioureas, wherein only $5 \mathrm{~mol} \%$ 
Table 1. Isolated guanidine yields

\begin{tabular}{|c|c|c|c|c|}
\hline 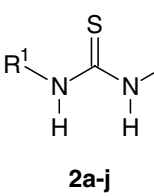 & $\begin{array}{c}\mathrm{H}_{-\mathrm{N}^{-}} \mathrm{R}^{3} \\
\mathrm{R}^{4} \\
\\
\text { 3a-d }\end{array}$ & $\begin{array}{l}\mathrm{Bil}_{3}(5 \mathrm{~mol} \%) \\
\mathrm{NaBiO}_{3}, \mathrm{Et}_{3} \mathrm{~N} \\
\mathrm{CH}_{3} \mathrm{CN}, \text { reflux }\end{array}$ & $\mathrm{H}^{-1}$ & $R^{2}$ \\
\hline Guanidine & Thiourea $\mathrm{R}^{1} / \mathrm{R}^{2}$ & Amine $\mathrm{R}^{3} / \mathrm{R}^{4}$ & $\begin{array}{l}\text { Yield } \\
(\%)\end{array}$ & $\begin{array}{l}\text { Time } \\
\text { (h) }\end{array}$ \\
\hline $4 a$ & $\mathrm{Bz} / p-\mathrm{CH}_{3} \mathrm{OPh}$ & $p-\mathrm{CH}_{3} \mathrm{OPh} / \mathrm{H}$ & 92 & 10 \\
\hline $4 \mathrm{~b}$ & $\mathrm{Bz} / \mathrm{Ph}$ & $p-\mathrm{CH}_{3} \mathrm{OPh} / \mathrm{H}$ & 75 & 12 \\
\hline $4 c$ & $\mathrm{Bz} / p-\mathrm{CH}_{3} \mathrm{OPh}$ & $c-\mathrm{C}_{6} \mathrm{H}_{11} / \mathrm{H}$ & 86 & 3 \\
\hline 4d & $\mathrm{Bz} / p-\mathrm{CH}_{3} \mathrm{Ph}$ & $c-\mathrm{C}_{6} \mathrm{H}_{11} / \mathrm{H}$ & 65 & 8 \\
\hline $4 e$ & $\mathrm{Bz} / \mathrm{Ph}$ & $c-\mathrm{C}_{6} \mathrm{H}_{11} / \mathrm{H}$ & 77 & 3 \\
\hline $4 f$ & $\mathrm{Bz} / c-\mathrm{C}_{6} \mathrm{H}_{11}$ & $c-\mathrm{C}_{6} \mathrm{H}_{11} / \mathrm{H}$ & 69 & 21 \\
\hline $4 g$ & $\mathrm{Bz} / o-\mathrm{ClPh}$ & $c-\mathrm{C}_{6} \mathrm{H}_{11} / c-\mathrm{C}_{6} \mathrm{H}_{11}$ & 91 & 11 \\
\hline $4 h$ & $\mathrm{Bz} / p-\mathrm{CH}_{3} \mathrm{OPh}$ & $\mathrm{CH}_{2} \mathrm{CH}_{2} \mathrm{OCH}_{2} \mathrm{CH}_{2}$ & 97 & 5 \\
\hline $4 i$ & $\mathrm{Ph} / p-\mathrm{CH}_{3} \mathrm{OPh}$ & $\mathrm{CH}_{2} \mathrm{CH}_{2} \mathrm{OCH}_{2} \mathrm{CH}_{2}$ & 95 & 3 \\
\hline $4 j$ & $\mathrm{Ph} / c-\mathrm{C}_{6} \mathrm{H}_{11}$ & $c-\mathrm{C}_{6} \mathrm{H}_{11} / \mathrm{H}$ & 95 & 7 \\
\hline
\end{tabular}

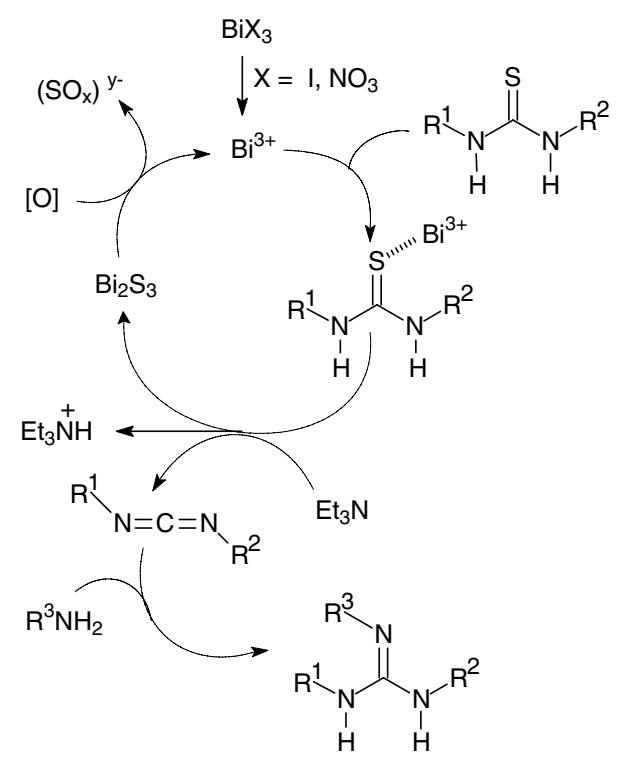

Figure 1. Proposed catalytic cycle for the Bi(III)-promoted guanylation of thioureas.

of inorganic thiophiles and no excess of amine was required, with yields and scope comparable with the stoichiometric $\mathrm{HgCl}_{2}$ protocol. ${ }^{6}$ Efforts are underway to elucidate the mechanistic details of this reaction and define the scope, limitations and synthetic applications to natural and unnatural bioactive guanidines.

\section{Acknowledgements}

The authors thank CNPq and FAPESB for support and fellowships, and Professor A.C.S. Costa for donation of $\mathrm{BiI}_{3}$.

\section{References and notes}

1. Review on synthesis: Manimala, J. C.; Anslyn, E. Eur. J. Org. Chem. 2002, 3909-3922; Representative papers: (a) Katritzky, A.; Rogovoy, B. V.; Cai, X.; Kirichenko, N.; Kovalenko, K. V. J. Org. Chem. 2004, 69, 309-313; (b) Gers, T.; Kunce, D.; Markowski, P.; Izdebski, J. Synthesis 2004, 37-42; (c) Miller, C. A.; Batey, R. A. Org. Lett. 2004, 6, 699-702; (d) Bowser, A. M.; Madalengoitia, J. S. Org. Lett. 2004, 6, 3409-3412; (e) Porcheddu, A.; Giacomelli, G.; Chighine, A.; Masala, S. Org. Lett. 2004, 6, 4925-4927.

2. (a) Organobismuth Chemistry; Suzuki, H., Matano, Y., Eds.; Elsevier: Amsterdam, 2001; (b) Leonard, N. M.; Wieland, L. C.; Mohan, R. S. Tetrahedron 2002, 58, 8373-8397.

3. (a) Evans, P. A.; Andrews, W. J. Tetrahedron Lett. 2005, 46, 5625-5627; (b) Srivastava, N.; Banik, B. K. J. Org. Chem. 2003, 68, 2109-2114.

4. (a) Cunha, S.; Lima, B. R.; Souza, A. R. Tetrahedron Lett. 2002, 43, 49-52; (b) Cunha, S.; Rodrigues, M. T., Jr.; Silva, C. C.; Napolitano, H. B.; Vencato, I.; Lariucci, C. Tetrahedron 2005, 61, 10536-10540.

5. The use of $\mathrm{NaBiO}_{3}$ in organic transformations is scarcely reported. For reviews, see Refs. 2a,2b.

6. The general synthetic procedure is as follows: to a solution of $0.5 \mathrm{mmol}$ of thiourea in $7 \mathrm{~mL}$ of $\mathrm{CH}_{3} \mathrm{CN}$ were added $0.5 \mathrm{mmol}$ of amine and $1 \mathrm{mmol}$ of $\mathrm{Et}_{3} \mathrm{~N}$, and then $0.5 \mathrm{mmol}$ of $\mathrm{NaBiO}_{3}$ and $0.025 \mathrm{mmol}$ of $\mathrm{BiI}_{3}$ were added to the solution with vigorous magnetic stirring. The suspension was left stirring for $10 \mathrm{~min}$ at room temperature and became black. After this time, it was heated at reflux at the indicated time in Table 1. The solvent was evaporated and $20 \mathrm{~mL}$ of $\mathrm{CH}_{2} \mathrm{Cl}_{2}$ was added. The suspension was filtered through a pad of Celite and the pad washed with $10 \mathrm{~mL}$ of $\mathrm{CH}_{2} \mathrm{Cl}_{2}$. The filtrate was dried over anhydrous $\mathrm{MgSO}_{4}$, filtrated and the solvent was evaporated. The crude residue was recrystallized from $\mathrm{Et}_{2} \mathrm{O}$ /petroleum ether affording a solid or a viscous oleo as indicated in each case.

Compound 4d: $\mathrm{Mp} 100-103{ }^{\circ} \mathrm{C}$. IR (KBr): $3287,1570 \mathrm{~cm}^{-1}$. ${ }^{1} \mathrm{H}$ NMR $\left(\mathrm{CDCl}_{3}\right): 1.10-2.10(10 \mathrm{H}, \mathrm{m}) ; 2.34(3 \mathrm{H}, \mathrm{s}) ; 4.15$ $(1 \mathrm{H}, \mathrm{m}) ; 4.80(1 \mathrm{H}, \mathrm{br}) ; 7.13(2 \mathrm{H}, \mathrm{d}, J=8.0 \mathrm{~Hz}) ; 7.21(2 \mathrm{H}, \mathrm{d}$, $J=8.0 \mathrm{~Hz}) ; 7.38-7.45(3 \mathrm{H}, \mathrm{m}) ; 8.24(2 \mathrm{H}, \mathrm{d}, J=6.3 \mathrm{~Hz})$; $11.97(1 \mathrm{H}, \mathrm{br}) .{ }^{13} \mathrm{C}$ NMR $\left(\mathrm{CDCl}_{3}\right): 20.9\left(\mathrm{CH}_{3}\right) ; 24.7\left(\mathrm{CH}_{2}\right)$; $25.5\left(\mathrm{CH}_{2}\right) ; 33.1\left(\mathrm{CH}_{2}\right) 50.0(\mathrm{CH}) ; 125.3(\mathrm{CH}) ; 127.7(\mathrm{CH})$; $129.0(\mathrm{CH}) ; 130.9(\mathrm{CH}) ; 133.4(\mathrm{C}) ; 136.6(\mathrm{C}) ; 138.8(\mathrm{C})$; 158.0 (C); $177.3(\mathrm{C})$.

Compound 4e: $\mathrm{Mp} 107-110^{\circ} \mathrm{C}$. IR (KBr): $3250,1570 \mathrm{~cm}^{-1}$. ${ }^{1} \mathrm{H}$ NMR $\left(\mathrm{CDCl}_{3}\right): 1.10-2.15(10 \mathrm{H}, \mathrm{m}) ; 4.18(1 \mathrm{H}, \mathrm{m}) ; 4.84$ $(1 \mathrm{H}, \mathrm{br}) ; 7.20-7.30(3 \mathrm{H}, \mathrm{m}) ; 7.35-7.50(5 \mathrm{H}, \mathrm{m}) ; 8.26(2 \mathrm{H}, \mathrm{d}$, $J=6.6 \mathrm{~Hz}) ; 12.97(1 \mathrm{H}, \mathrm{br}) \cdot{ }^{13} \mathrm{C} \mathrm{NMR}\left(\mathrm{CDCl}_{3}\right): 24.7\left(\mathrm{CH}_{2}\right)$; $25.4\left(\mathrm{CH}_{2}\right) .33 .0\left(\mathrm{CH}_{2}\right) ; 50.1(\mathrm{CH}) ; 125.0(\mathrm{CH}): 126.5(\mathrm{CH})$; $127.8(\mathrm{CH}) ; 128.9(\mathrm{CH}) ; 129.9(\mathrm{CH}) ; 131.03(\mathrm{CH}) ; 136.2(\mathrm{C})$; 138.6 (C); 157.7 (C); 177.4 (C).

Compound 4g: $\mathrm{Mp} 125-127{ }^{\circ} \mathrm{C}$; IR (KBr): 3065, 1610, 1544, $1366 \mathrm{~cm}^{-1} .{ }^{1} \mathrm{H}$ NMR $\left(\mathrm{CDCl}_{3}\right): 1.00-2.25(20 \mathrm{H}, \mathrm{m}) ; 3.25-$ $3.40(2 \mathrm{H}, \mathrm{m}) ; 7.00-7.20(2 \mathrm{H}, \mathrm{m}) ; 7.30-7.50(2 \mathrm{H}, \mathrm{m}) ; 8.26(1 \mathrm{H}$, $\mathrm{d}, J=6.6 \mathrm{~Hz}) ; 11.07(1 \mathrm{H}, \mathrm{br}) \cdot{ }^{13} \mathrm{C}$ NMR $\left(\mathrm{CDCl}_{3}\right): 25.5$ $\left(\mathrm{CH}_{2}\right) ; 26.3\left(\mathrm{CH}_{2}\right) ; 31.1\left(\mathrm{CH}_{2}\right) ; 58.6(\mathrm{CH}) ; 125.2(\mathrm{CH}) ; 126.0$ $(\mathrm{CH}) ; 127.2(\mathrm{CH}) ; 127.2(\mathrm{CH}) ; 127.8(\mathrm{CH}) ; 128.7(\mathrm{C}) ; 129.0$ $(\mathrm{CH}) ; 130.0(\mathrm{CH}) ; 130.9(\mathrm{CH}) ; 138.5(\mathrm{C}) ; 160.7(\mathrm{C}) ; 175.4(\mathrm{C})$. Compound 4h: oil. IR (film): $3013,1600,1575 \mathrm{~cm}^{-1} .{ }^{1} \mathrm{H}$ NMR $\left(\mathrm{CDCl}_{3}\right): 3.55(4 \mathrm{H}, \mathrm{m}) ; 3.67(4 \mathrm{H}, \mathrm{m}) ; 3.78(3 \mathrm{H}, \mathrm{s}) ; 6.85$ $(2 \mathrm{H}, \mathrm{d}, J=8.8 \mathrm{~Hz}) ; 7.06(2 \mathrm{H}, \mathrm{d}, J=8.8 \mathrm{~Hz}) ; 7.40-7.55(3 \mathrm{H}$, $\mathrm{m}) ; 8.22(2 \mathrm{H}, \quad \mathrm{d}, \quad J=6.9 \mathrm{~Hz}) ; 11.85 \quad(1 \mathrm{H}, \quad$ br $) .{ }^{13} \mathrm{C}$ $\operatorname{NMR}\left(\mathrm{CDCl}_{3}\right): 46.9\left(\mathrm{CH}_{2}\right) ; 55.4\left(\mathrm{CH}_{3}\right) ; 66.3\left(\mathrm{CH}_{2}\right) ; 114.8$ $(\mathrm{CH}) ; 123.4(\mathrm{CH}) ; 127.9(\mathrm{CH}) ; 129.2(\mathrm{CH}) ; 131.5(\mathrm{CH}) ; 132.3$ (C); 138.2 (C); 156.9 (C); 160.2 (C); 177.1 (C). 